\title{
Space for all
}

\section{The exploration of the Solar System is an expensive endeavour. The greater the number of nations that engage in peaceful planetary research the better.}

In a rapidly changing and uncertain economic climate, tough choices will have to be made. Just a month or so ago, research on Titan took a blow when a proposal to NASA for a mission to this fascinating moon of Saturn was beaten by a proposal for exploring the icy Jovian satellite Europa. The European Space Agency (ESA) will probably undertake a parallel mission to Ganymede, another body in Jupiter's realm.

For now, it seems that the world's two premier exploration agencies do not have the money for additional missions, and the future of the Russian space program is unclear. However, the entry of nations such as China, Japan and India into space exploration has expanded the options. No more will the world have to rely solely on the space veterans to satisfy its curiosity about Earth's neighbours in the Solar System.

Even Iran took a first step towards the development of a functional space programme by successfully launching a tiny satellite. Iran itself naturally took particular pride in this achievement, but western media have been quick to highlight the potential military and political implications of the launch. Five decades after the launch of the Soviet satellite Sputnik, it seems that space, nationalism and geopolitics are just as intertwined with each other today as they were then.

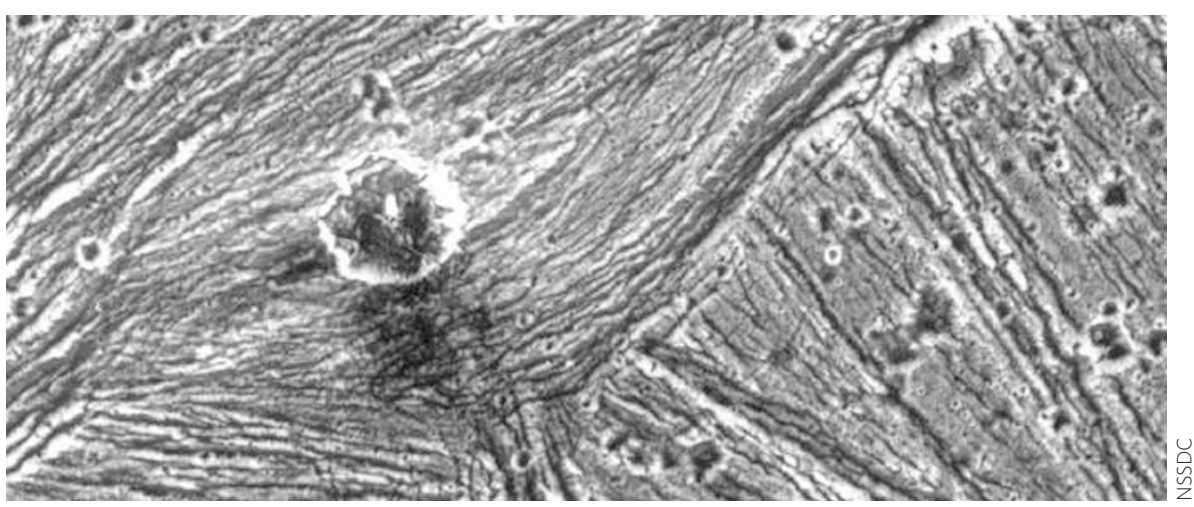

A Galileo image of part of the Uruk Sulcus region on Jupiter's largest satellite, Ganymede, taken on 27 June 1996. The European Space Agency (ESA) is hoping to send a probe to Ganymede in 2020.

The entry of more players and their desire to explore is an important and positive development: the new entrants have burgeoning economies and no shortage of scientific talent, and their space programmes are already injecting new life into the study of the Moon (see Feature on page 234). Because of its close proximity to Earth, the Moon is an ideal platform for newcomers in the space game to perfect the art (and science!) of building and launching spacecraft and probes. In the long-run, this may allow NASA and ESA to focus more on far-away planetary bodies.
Of course, more missions from a larger number of nations will require greater international cooperation. This is an opportunity as well as a challenge. The signs are good: India's Chandrayaan-1 spacecraft carries scientific instruments from the US and Europe.

As the recent collision between two satellites suggests, space is getting crowded. Nevertheless, the scientific benefits from a more diverse field of space-going nations are likely to outweigh the costs.

\section{Farewell to presubs}

\section{Presubmission enquiries have only rarely resolved the question of whether a paper is suitable for Nature Geoscience. We are now removing this option from our online submission system.}

Until now, the online submission system for Nature Geoscience has allowed prospective authors to contact the editors formally with a 'presubmission enquiry', consisting of an abstract and a brief cover letter. A small fraction of our authors have taken up this option, which promises a fast evaluation of the suitability in principle of a piece of research for publication in Nature Geoscience. We are now closing down this option.

Presubmission enquiries are useful in fields where the interest of a manuscript is readily apparent from a brief summary. We have found that in the geosciences, such a shortcut assessment is almost always impossible: the novelty and importance of a study usually relies on factors that can only be fully appreciated by reading the whole paper and often previous publications in the field.

We would hate to miss an interesting paper because of a badly written abstract. In response to a large proportion of presubmission enquiries we have therefore simply asked to see the full manuscript.
Furthermore, we have always carefully considered full submissions of manuscripts that had previously received a discouraging response on a presubmission enquiry.

We feel that our authors are best served by a careful assessment of the suitability of their paper, based on all the necessary information, therefore we now encourage authors to submit their full manuscripts right away. Of course, for any brief and informal enquiries - regarding a manuscript submission or anything else - we can always be contacted at geoscience@nature.com. 